\title{
A Low-Makeup Beveled Tip Capillary Electrophoresis /Electrospray Ionization Mass Spectrometry Interface for Micellar Electrokinetic Chromatography and Nonvolatile Buffer Capillary Electrophoresis
}

\author{
Mei-Chun Tseng, Yet-Ran Chen, and Guor-Rong Her* \\ Department of Chemistry, National Taiwan University, Taipei, Taiwan, R.O.C
}

A robust interface has been developed for interfacing micellar electrokinetic chromatography (MEKC) and nonvolatile buffer capillary electrophoresis (CE) to electrospray ionization mass spectrometry (ESI-MS). The interface consists of two parallel capillaries for separation (50 $\mu \mathrm{m}$ i.d. $\times 155 \mu \mathrm{m}$ o.d.) and makeup (50 $\mu \mathrm{m}$ i.d. $\times 155$ $\mu \mathrm{m}$ o.d.) housed within a larger capillary (530 $\mu \mathrm{m}$ i.d. $\times$ $690 \mu \mathrm{m}$ o.d.). The capillaries terminate in a single tapered tip having a beveled edge. The use of a tapered beveled edge results in a greater tip orifice diameter $(75 \mu \mathrm{m})$ than in a previous design from our laboratory $(25 \mu \mathrm{m})$ that used a flat tip. While maintaining a similar optimum flow rate and consequently similar sample dilution, a $75-\mu \mathrm{m}$ beveled emitter is more rugged than a $25-\mu \mathrm{m}$ flat tip. Furthermore, the incorporation of a sheath liquid capillary allows the compositions of the final spray solution to be controlled. The application of this novel CE/ESI-MS interface was demonstrated for MEKC using mixtures of triazines (positive ion mode) and phenols (negative ion mode). The ability to perform CE/ESI-MS using a nonvolatile buffer was demonstrated by the analysis of gangliosides with a buffer consisting of $\mathbf{4 0} \mathrm{mM}$ borate and 20 mM $\alpha$-cyclodextrin.

On-line coupling of capillary electrophosesis (CE) with electrospray ionization mass spectrometry (ESI-MS) is an attractive tool for direct identification of analytes because it combines excellent selectivity with structural confirmation by MS or MS/ MS. Among the techniques used to couple CE to ESI-MS, the sheath liquid, ${ }^{1-3}$ sheathless, ${ }^{4-13}$ and liquid junction ${ }^{14-16}$ interfaces

* To whom correspondence should be addressed. Tel: (886) 2-23690152 ext. 109. Fax: (886) 2-23638058. E-mail:grher@ntu.edu.tw.

(1) Smith, R. D.; Barinaga, C. J.; Udseth, H. R.Anal. Chem. 1988, 60, 19481952.

(2) Chu, Y. H.; Dunayevskiy, Y. M.; Kirby, D. P.; Vouros, P.; Karger, B. L. J. Am. Chem. Soc. 1996, 118, 7827-7835.

(3) Nashabeh, W.; Greve, K. F.; Kirby, D.; Foret, F.; Karger, B. L.; Reifsnyder, D. H.; Builder, S. E. Anal. Chem. 1994, 66, 2148-2154.

(4) Whal, J. H.; Gale, D. C.; Smith, R. D. J. Chromatogr. 1994, 659, 217-222.

(5) Kriger, M. S.; Cook, K. D.; Ramsey, R. S. Anal. Chem. 1995, 67, 385-389.

(6) M oini, M. Anal. Chem. 2001, 73, 3497-3501.

(7) Chang, Y. Z.; Her, G. R. Anal. Chem. 2000, 72, 626-630. are the most widely used. Of these, the sheath liquid interface is the most popular because of robustness and ease of use. In addition to providing electrical contact with the outlet end of the separation capillary, a liquid sheath allows CE/ ESI-M S operation with a wide range of buffer systems. ${ }^{1,2}$ However, the flow rate of sheath liquid, in the range of several microliters per minute, is significantly higher than the flow rate of CE. Unfortunately, the associated dilution of the CE effluent ultimately reduces ESI-M S sensitivity.

In sheathless interfaces, the electrical connection to the capillary outlet is achieved by direct metal/liquid contact at or near the tip of the CE capillary outlet. ${ }^{4-13}$ Because there is no sheath liquid to dilute the CE effluent, no dilution of the sample occurs prior to the mass spectrometer and maximum ESI sensitivity is obtained. However, the use of a single capillary in sheathless CE/ESI-M S interface causes several practical issues. For example, it is often difficult to find a solution optimized for both CE separation and ES ionization efficiency. Therefore, the selection of the $C E$ running buffer is limited.

Given the limitations of the two interfaces, a more ideal interface would combine the versatility of the sheath liquid design with the sensitivity of the sheathless format. Recently, we developed a low-flow sheath liquid interface using a sprayer having an orifice of reduced diameter. ${ }^{17} \mathrm{~A}$ smaller spray orifice was used because it is known that optimum flow rate decreases with orifice

(8) Chang, Y. Z.; Chen, Y. R.; Her, G. R. Anal. Chem. 2001, 73, 5083-5087.

(9) Chen, Y. R., Her, G. R. Rapid Commun. Mass Spectrom. 2003, 17, 437441.

(10) Wetterhall, M ., Nilsson, S., M arkides, K. E., Bergquist, J. Anal. Chem. 2002, $74,239-245$.

(11) Petersson, M. A., Hulthe, G., Fogelqvist, E. J. Chromatogr., A 1999, 854, $141-154$.

(12) Barroso, M. B., de Jong, A. P. J. Am. Soc. Mass Spectrom. 1999, 10, 12711278.

(13) Janini, G. M., Conrads, T. P., Wilkens, K. L., Issaq, H. J., Veenstra, T. D.Anal. Chem. 2003, 75, 1615-1619.

(14) Lee, E. D.; M uck, W.; Henion, J. D.; Covey, T. D. Biomed. Environ. Mass Spectrom. 1989, 18, 844-850.

(15) von Brocke, A.; Nicholson, G.; Bayer, E. Electrophoresis 2001, 22, 12511266.

(16) Ding, J.; Vouros, P. Anal. Chem. 1999, 71, 378A-385A.

(17) Chen, Y. R.; Tseng, M. C.; Chang, Y. Z.; Her, G. R.Anal. Chem. 2003, 75, 503-508.

10.1021/ac049330i CCC: $\$ 27.50$ @ 2004 American Chemical Society Published on Web 09/30/2004 
diameter. The advantage of the smaller orifice sprayer was that it lowered the operational flow rate and thus reduced the sample dilution. Improved sensitivity of the low-flow interface was achieved when compared to a conventional sheath liquid interface. ${ }^{17}$

A further advantage of a smaller orifice sprayer is that it reduces the size of the ESI droplet. Smaller droplets have a higher surface-to-volume ratio, which results in increased sensitivity and a higher tolerance to salts in buffers. ${ }^{18}$ Consequently, the lowflow sheath liquid interface described previously provided better performance than a conventional sheath liquid interface when coupling micellar electrokinetic chromatography (MEKC) to ESI$\mathrm{MS}^{17}$

Despite the success of the low-flow interface, it was found to be incompatible with the nonvolatile buffers used for CZE/ESIMS applications. In particular, the initial flat tip design was found to be prone to clogging when used in CE/ESI-M S applications involving nonvolatile buffers.

In this paper, an improved version of the low-makeup interface is reported in which both the separation and sheath liquid capillaries are tapered and terminated in a common tip having a beveled edge. The use of a beveled tip allows the use of a tip with significantly larger orifice and thus the tip is less likely to be clogged. Another modification of the interface was the introduction of a makeup flow capillary in the interface. The use of a makeup flow column makes the final spray solution much easier to control. Successful applications are presented that demonstrate the ability of this new interface to be used with M EKC as well as conventional CE using nonvolatile buffers.

\section{EXPERIMENTAL SECTION}

Reagents. Triazine standards were obtained from Supelco (Bellefonte, PA). 2,4,-Dinitrophenol was obtained from Janssen. 4-N itrophenol, 4-chloro-3-methylphenol, 2,4-dichlorophenol, 2,4dimethylphenol, 2-methyl-4,6-dinitrophenol (2-M-4,6-DNP), and 2,4,6-trichlorophenol were purchased from Fluka. Type III gangliosides (GM 1, GD 1a, GD 1b, and GT1b purified from bovine brain containing $\sim 20 \% \mathrm{~N}$-acetylneuraminic acid), 2-(N-cyclohexylamino) ethanesulfonic acid (CHES), and sodium dodecyl sulfate (SDS) were obtained from Sigma (St. Louis, M O). Ammonium acetate, methanol, and 2-propanol of HPLC grade were purchased from J. T. Baker (Phillipsburg, NJ) and used without further purification. $\alpha$-Cyclodextrin $(\alpha-C D)$ was obtained from Nacalai Tesque. Hydrofluoric acid ( $>48 \%$ ) was obtained from Ferak Berlin. Anhydrous sodium tetraborate and ammonium hydroxide were obtained from Janssen. Deionized water (M illi-Q water system, M illipore Inc., Bedford, M A) was used in the preparation of the samples and buffer solution.

Fabrication of the Low-Flow Beveled Tip CE/ESI-MS Interface. The interface is shown in Figure 1. A beveled tapered tip was connected to a liquid reservoir (a microcentrifuge tube) and used as a sprayer for ESI. Two capillaries were inserted into the sprayer. One was used as the separation column, and the other was used to deliver a makeup flow. A syringe pump (Cole-Parmer, Vernon Hill, IL) was used to control the flow rate of the makeup flow. In this interface, the electrical contact is achieved by inserting

(18) Juraschek, R.; Dulcks, T.; Karas, M. J. Am. Soc. Mass Spectrom. 1999, 10, $300-308$. a platinum wire into the liquid reservoir. This electrode acts both as the electrode of the CE electric circuit and as a connection for the electrospray ionization voltage.

The beveled tapered tip was fabricated using a 530- $\mu \mathrm{m}-\mathrm{i} . \mathrm{d}$. and 690- $\mu$ m-0.d. capillary (Restek). The capillary was drawn manually using a vertically suspended section of capillary to which a small weight $(45 \mathrm{~g})$ had been attached. The capillary was slowly heated to the melting stage using a butane/ oxygen microtorch (Pro-Iroda Industries Inc.) and then quickly withdrawn. A tip of $80-\mu \mathrm{m}$ 0.d. and $75-\mu \mathrm{m}$ i.d. was obtained by removing the end of the tip using a ceramic cutter aided by visual inspection with a microscope. The tip was then wrapped with cellophane adhesive tape and ground with a rasp to $\sim 45^{\circ}$. After grinding, the tape was removed from the tip before being connected to a microcentrifuge tube.

Preparation of the Separation and Makeup Flow Capillaries. In this design, both the separation and makeup columns are inserted into the sprayer (Figure 1). Because the inner diameter of the emitter is $530 \mu \mathrm{m}$, a capillary with $155-\mu \mathrm{m}$ o.d. was chosen as the separation as well as the makeup column. Furthermore, to minimize the dead volume between the capillaries and the sprayer, the separation and makeup columns were both tapered. To prepare the tapered capillaries, the capillaries were drawn manually using a vertically suspended section of capillary to which a small weight $(10 \mathrm{~g})$ had been attached. The capillaries were slowly heated to the melting stage and then quickly withdrawn. The tapered capillaries were cut to $55 \mu \mathrm{m}$ o.d. $\times 35$ $\mu \mathrm{m}$ i.d. and $30 \mu \mathrm{m}$ o.d. $\times 15 \mu \mathrm{m}$ i.d. and then etched by immersing in $48 \% \mathrm{HF}$ for 10 and $5 \mathrm{~min}$, respectively. After etching, the dimensions of capillaries were about $50 \mu \mathrm{m}$ o.d. $\times 45 \mu \mathrm{m}$ i.d. and $25 \mu \mathrm{m}$ o.d. $\times 20 \mu \mathrm{m}$ i.d.. The capillary dimension was estimated by comparing with a $50-\mu \mathrm{m}$-i.d. capillary under the microscope. The capillaries were washed with deionized water to remove remaining $\mathrm{HF}$. B efore use, the separation capillary was conditioned with $1 \mathrm{M} \mathrm{NaOH}$, following with $0.1 \mathrm{M} \mathrm{NaOH}$ and deionizied water.

In this interface, running buffer was driven by electroosmotic flow of the capillary, whereas the flow rate of makeup liquid was controlled by a syringe pump. In addition to the flows from separation and makeup column, there was also a minor flow from the reservoir. To estimate the flow rate from the reservoir, three compounds with similar ionization efficiency (prometon, ametryn, terbutyne) were added into the running buffer, makeup column, and reservoir. The flow rate of the makeup column was controlled at 200 and $400 \mathrm{~nL} / \mathrm{min}$, respectively. Based on the relative intensity of the analytes, the flow rate from the reservoir was estimated to be $\sim 40 \mathrm{~nL} / \mathrm{min}$ at a makeup liquid of 200 and $400 \mathrm{~nL} / \mathrm{min}$.

CE Instrument. The CE instrument was configured in-house. B riefly, the setup consisted of a $1000 \mathrm{R}$ high-voltage power supply (Spellman, Plainview, NY) connected to a platinum electrode in a vial containing CE buffer and operated at constant-voltage mode. One end of the separation capillary was inserted into the CE buffer, and the other end was inserted into the CE/ESI-M S interface. In a CE/UV study, the detector (UV-C, Rainin, Emeryville, CA) wavelength was set to $200 \mathrm{~nm}$.

ESI-MS. All mass spectrometry experiments were conducted on a LCQ ion trap mass spectrometer (Finnigan M AT, San Jose, CA), and the CE/ESI-M S electropherograms were acquired in selected ion monitoring mode (SIM). A commercial $x-y-z$ 

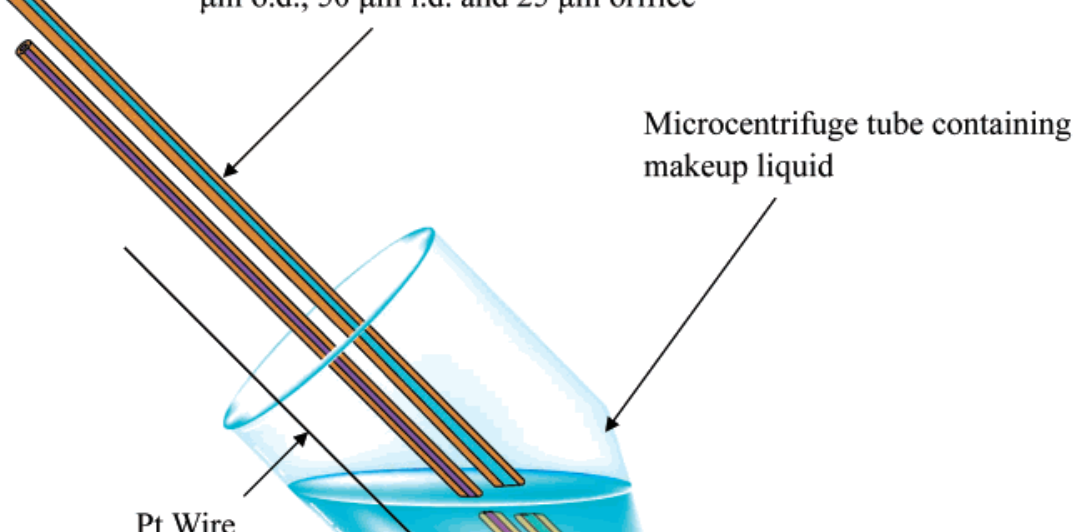

Pt Wire

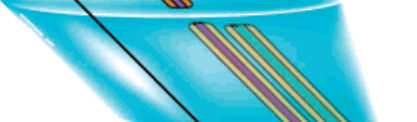

Wide bore column with $530 \mu \mathrm{m}$ i.d. and $75 \mu \mathrm{m}$ beveled tip orifice

CE separation capillary with $155 \mu \mathrm{m}$ o.d., $50 \mu \mathrm{m}$ i.d. and $50 \mu \mathrm{m}$ orifice
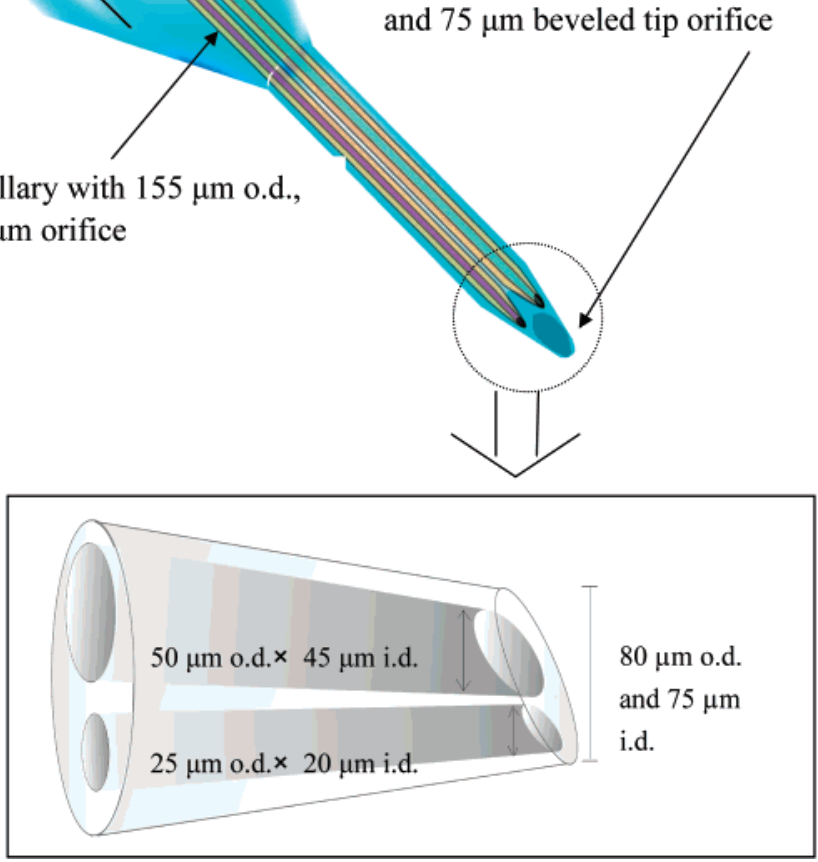

Figure 1. Schematic representation of the low-makeup beveled tip CE/ESI-MS interface.

translation stage for LCQ API source (Protana Co., Odense, Denmark) was used for mounting the low-makeup sheath liquid CE/ESI-MS interface. The position of the interface could be adjusted via the micrometer screws of the translation stage. A nebulizing gas was not necessary, and the heating capillary was kept at a temperature of $200{ }^{\circ} \mathrm{C}$. A high-voltage power supply (Glassman, Whitehouse Station, NJ) with 0-30-kV range, was used to apply voltage across the capillary columns.

Conventional Sheath Liquid CE/ESI-MS Interface. The CE/ M S interface equipped with the LCQ ion trap mass spectrometer (Finnigan MAT) was used for all the sheath liquid experiments. The interface utilizes a triaxial flow arrangement whereby CE effluent is mixed with a suitable sheath liquid at the tip and nebulized by nitrogen gas. The sheath liquid was delivered at a flow rate of $5 \mu \mathrm{L} / \mathrm{min}$ by a syringe pump, and the flow rate of nebulization gas was set to 20 (an arbitrary unit).

MEKC/ESI-MS Analysis of Triazines. The M EKC running buffers contained $20 \mathrm{mM}$ ammonium acetate and $25 \mathrm{mM}$ SDS in water. The $\mathrm{pH}$ of the solution was adjusted to 7.0 with ammonia. The solution of triazine mixture ( $5 \mathrm{ppm}$ ) was hydrodynamically injected to the capillary using a 10 mbar pressure differential for a duration of $10 \mathrm{~s}$. The separation capillary was $70 \mathrm{~cm}$ in total length. The makeup liquid consisted of methanol, water, and acetic acid (70:30:1, v/ v/ v) and was injected into the liquid reservoir of the emitter and the syringe pump prior to CE/ MS analysis. The mass spectrometer was operated in positive ion mode, and data were collected in SIM mode.

MEKC/ ESI-MS Analysis of Phenols. The separation buffer contained $20 \mathrm{mM} \mathrm{CHES}$ and $40 \mathrm{mM}$ SDS in water. The $\mathrm{pH}$ of the solution was adjusted to 7.0 with ammonia. The separation capillary was $100 \mathrm{~cm}$ in total length. Before each run, the capillary was flushed with the separation buffer and equilibrated for $15 \mathrm{~min}$. The 20 ppm phenolic mixture was hydrodynamically injected onto the capillary using a 10 mbar pressure differential for a duration of $10 \mathrm{~s}$. The mass spectrometer was operated in the negative ion mode; therefore, a basic makeup liquid was required to assist negative ion formation. The makeup liquid, consisting of 2-propanol, water, and ammonium hydroxide (80:20;1, v/ v/ v), was placed into the liquid reservoir of the emitter and the syringe pump. 
CZE / E SI-MS Analysis of Gangliosides Using a Nonvolatile

Buffer. The separation buffer contained $40 \mathrm{mM}$ borate and 20 $\mathrm{mM} \alpha-\mathrm{CD}$ in water. The $\mathrm{pH}$ of the solution was adjusted to 10.6 with ammonia. The separation capillary was $100 \mathrm{~cm}$ in total length. The type III ganglioside mixture $(4 \mathrm{mg} / \mathrm{mL}$ ) was hydrodynamically injected onto the capillary using a 10 mbar pressure differential for a duration of $10 \mathrm{~s}$. The makeup liquid consisted of 2-propanol, water, and ammonium hydroxide (80:20:1, v/ v/ v). The mass spectrometer was operated in negative ion mode, and the data were collected in SIM mode

\section{RESULTS AND DISCUSSION}

Shape and Dimensions of the Interface. In our recently reported low-flow CE/ M S interface, a tip with a $25-\mu \mathrm{m}$ orifice was used as the sprayer in the CE/MS interface. ${ }^{17}$ B ecause the size of the emitter was considerably smaller than that of a conventional sheath liquid interface ( $\sim 400 \mu \mathrm{m})$, the optimum flow rate of the emitter is significantly lower than that of a conventional sheath liquid interface. Because of reduced sample dilution resulting from a lower optimum flow rate, the sensitivity of the interface was found to be better than that obtained with a conventional sheath liquid interface.

In the current interface design, instead of using a $25-\mu \mathrm{m}$ flat tip, ${ }^{17}$ a $75-\mu \mathrm{m}$ beveled tapered sprayer is used as the emitter. Although the orifice of a $75-\mu \mathrm{m}$ beveled tapered sprayer is significantly larger than the orifice of a $25-\mu \mathrm{m}$ flat tip, the optimum flow rate of the two emitters is expected to be similar. In a sheathless interface, we have shown that a $75-\mu \mathrm{m}$ beveled sprayer has anoptimum flow rate similar to that of a $25-\mu \mathrm{m}$ flat tip (2001000 and $200-500 \mathrm{~nL} / \mathrm{min}$ for $75-\mu \mathrm{m}$ beveled and $25-\mu \mathrm{m}$ blunt sprayer, respectively). ${ }^{8}$ While maintaining a similar optimum flow rate and consequently similar sample dilution, a $75-\mu \mathrm{m}$ beveled emitter is more rugged and durable than a $25-\mu \mathrm{m}$ flat tip because of the larger inner and outer diameters.

A second modification of the interface was the addition of a capillary to deliver makeup liquid. The flow rate of the makeup liquid was controlled by a syringe pump granting the final spray solution much easier to be controlled. Although capillary column of $360-\mu \mathrm{m}$ o.d. is the most popular column used in CE applications, to insert two capillaries (one separation, one makeup) into the sprayer (530- $\mu \mathrm{m} \mathrm{i.d.),} \mathrm{a} \mathrm{column} \mathrm{with} 155-\mu \mathrm{m}$ o.d. was chosen as the separation and the makeup capillary. Furthermore, for an interface to avoid extracolumn band broadening caused by diffusion, the dead volume between the tip of CE column and the tip of the sprayer must be kept to a minimum. To achieve that, one end of the columns was tapered down to $\sim 50-\mu \mathrm{m}$ o.d. for separation capillary and $\sim 25 \mu \mathrm{m}$ o.d. for makeup capillary so that the columns could be inserted to the very end of the sprayer.

Performance of the Interface in MEKC/ESI-MS. MEKC is known for its excellent resolving power in the separation of charged as well as neutral compounds. However, the surfactants such as SDS are known to cause ionization suppression. It has been proposed that a smaller droplet has better sensitivity and higher resistance to ionization suppression. ${ }^{18}$ Although the size of the orifice in this interface is significantly larger than that of a $25-\mu \mathrm{m}$ flat tip, because of the similar flow rate, the diameter of the droplet produced in the interface is expected to be similar to that of a $25-\mu \mathrm{m}$ flat tip. To evaluate the performance of the interface

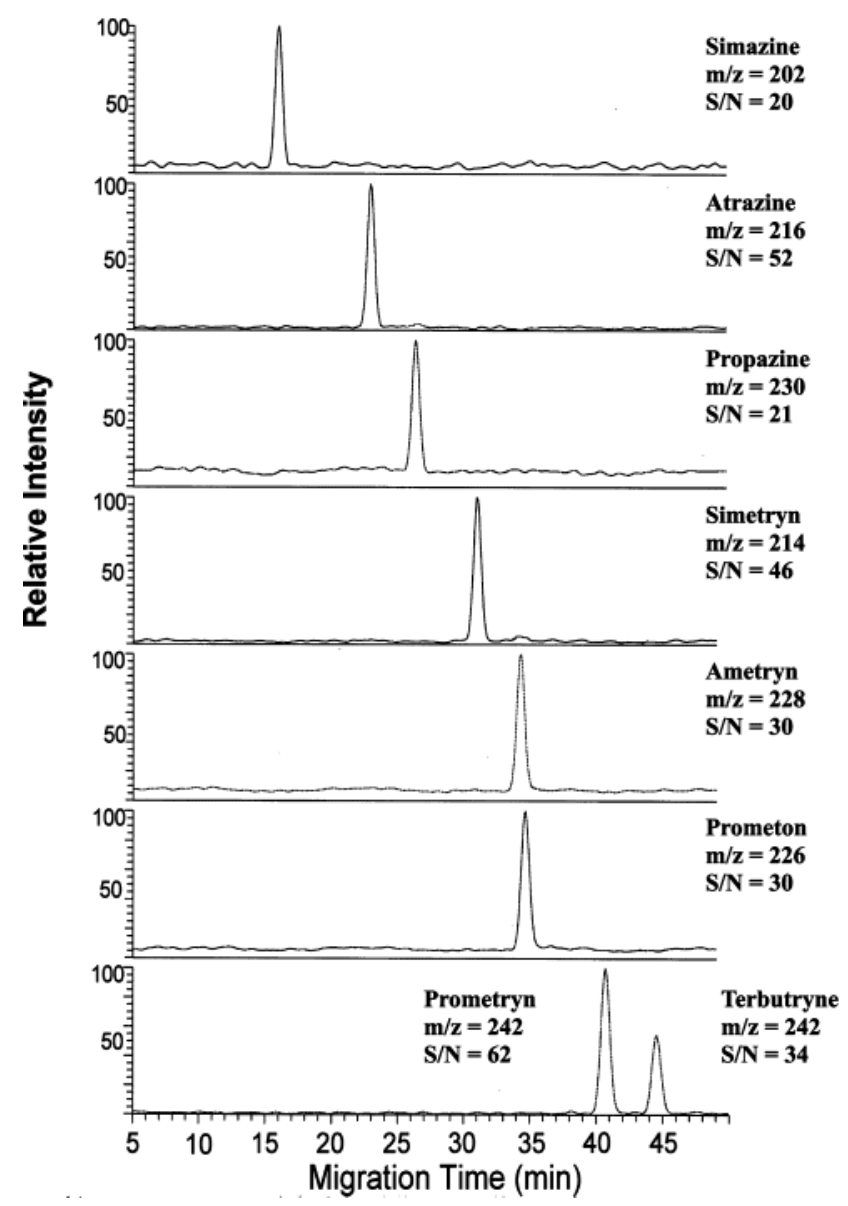

Figure 2. Mass electropherograms of $5 \mathrm{ppm}$ triazines using the low-makeup beveled tip interface in positive ion mode. The makeup liquid $(70 \%$ methanol with $1 \%$ acetic acid) was delivered by a syringe pump at a flow rate of $200 \mathrm{~nL} / \mathrm{min}$. The potential applied to the buffer reservoir was $+22 \mathrm{kV}$, and the $\mathrm{ESI}$ voltage was set at $+2 \mathrm{kV}$.

in the coupling of MEKC with ESI-M S using SDS as the surfactant, a $5 \mathrm{ppm}$ triazine mixture was hydrodynamically injected into the capillary using a $10 \mathrm{mbar}$ pressure differential for a duration of $10 \mathrm{~s}$. The MEKC running buffer was $20 \mathrm{mM}$ ammonium acetate and $25 \mathrm{mM}$ SDS in water at $\mathrm{pH}$ 7. The composition of the makeup liquid was critical to the performance of the CZE/ESI interface. ${ }^{19}$ Thus, makeup liquids of different composition were tested for sensitivity and stability under positive ESI conditions. A 1 ppm simetryn solution was electroosmotically infused through the silica capillary and then mixed with makeup liquid at the tip of the CE probe. The best result was obtained with a solution of 70:30 methanol/ water containing $1 \%$ acetic acid. To produce the best signal, the flow rate of the makeup liquid on analyte signals was also studied. The results showed that the highest signals were observed at a flow rate of $200 \mathrm{~nL} / \mathrm{min}$. The total flow rate of the interface was $\sim 390 \mathrm{nl} / \mathrm{min}$ ( $200 \mathrm{~nL} / \mathrm{min}$ from makeup column, $150 \mathrm{~nL} / \mathrm{min}$ from separation column, and $40 \mathrm{~nL} / \mathrm{min}$ from the reservoir), which was in the range of the optimum flow rate of a $75-\mu \mathrm{m}$ beveled sprayer (200-1000 nL/ min). Under the optimum conditions, the results of on-line M EKC/ESI-M S analysis of eight triazines were shown in Figure 2. Because the peak width was

(19) Foret, F.; Thompson, T. J.; Vouros, P.; Karger, B. L.; Gebauer, P.; Bocek, P. Anal. Chem. 1994, 66, 4450-4458. 
similar to the conventional interface, ${ }^{17}$ no significant band boarding caused by the dead volume was observed in this interface. Although the signal/ noise ratios in Figure 2 appeared to be similar to the data obtained with the previously reported low-flow flat tip interface, ${ }_{17}^{17}$ the concentration of the triazines used in this study was 4 -fold lower than that used in the low-flow interface. In the analysis of a $5 \mathrm{ppm}$ triazine mixture, the average $\mathrm{S} / \mathrm{N}$ ratios $(\mathrm{n}=$ 3) of the $75-\mu \mathrm{m}$ beveled and the $25-\mu \mathrm{m}$ flat tip interfaces were $39.8 \pm 13.6$ and $10.4 \pm 22.2 \%$ respectively. Therefore, better sensitivity was obtained in this interface in comparison with the low-flow interface. For the $75-\mu \mathrm{m}$ beveled interface, the LOD of triazines was found to be $\sim 1 \mathrm{ppm}$. One possible explanation for the improved sensitivity is that the flow rate of the makeup liquid and thus the composition of spray solution can be controlled precisely by a syringe pump, whereas in the low-flow flat tip interface, the flow rate of the makeup liquid is uncontrolled and could be affected by the buffer composition and the potential applied to the sprayer.

The durability of the $75-\mu \mathrm{m}$ beveled interface in MEKC-M S operation was studied by continuous infusion of a $1 \mathrm{ppm}$ prometon solution (in $25 \mathrm{mM}$ SDS and $20 \mathrm{mM}$ ammonium acetate), and the makeup liquid was supplied at $400 \mathrm{~nL} / \mathrm{min}$. The signal was found to be stable for $28 \mathrm{~h}$ and gradually decreased to $75 \%$ after $36 \mathrm{~h}$ of operation. However, for the $25-\mu \mathrm{m}$ tapered interface, the signal was stable only for $4 \mathrm{~h}$.

Under ESI-M S, the surfactant SDS gives rise to strong signals in both positive and negative ion modes. ${ }^{20}$ The ion suppression is expected to be more serious in the negative ion mode because the anionic surfactants would occupy the surface and compete with the analytes for the excess negative charge of the droplet. In an infusion experiment, atrazine and 2-M -4,6-D N P were used to study the response under positive and negative ESI using a low-flow interface. The results showed that, at a SDS concentration of $40 \mathrm{mM}$, the signals of the analyte dropped to $\sim 25 \%$ in positive ion mode and $\sim 8 \%$ in negative ion mode. To evaluate the performance of this interface in negative ion $M E K C$, a mixture of seven phenolic compounds (20 ppm) was hydrodynamically injected into the capillary using a $10 \mathrm{mbar}$ pressure differential for a duration of $10 \mathrm{~s}$. The separation buffer contained $20 \mathrm{mM}$ CHES and $40 \mathrm{mM}$ SDS in water at pH 7. The composition of the makeup liquid was studied, and the best result was obtained with a solution of 80:20 2-propanol/ water containing 1\%ammonia. The effect of flow rate of the makeup liquid on the signal intensity was also studied, and the best result was obtained at a makeup flow rate of $400 \mathrm{~nL} / \mathrm{min}$. The total flow was $\sim 590 \mathrm{~nL} / \min$ ( 400 $\mathrm{nL} / \mathrm{min}$ from makeup column, $150 \mathrm{~nL} / \mathrm{min}$ from separation column, and $40 \mathrm{~nL} / \mathrm{min}$ from the reservoir), which was in the range of the optimum flow rate of a $75-\mu \mathrm{m}$ beveled sprayer (200$1000 \mathrm{~nL} / \mathrm{min})$. Figure 3 shows the mass electropherograms of phenols using the low-flow beveled tip interface at a makeup flow rate of $400 \mathrm{~nL} / \mathrm{min}$. The quality of the signals was found to be better than that obtained with the recently reported low-flow flat tip interface in which four of the seven phenols were not detected (data not shown).

(20) Smith, R. D.; Barinaga, C. J.; Udseth, H. R.Anal. Chem. 1988, 60, 19481952.

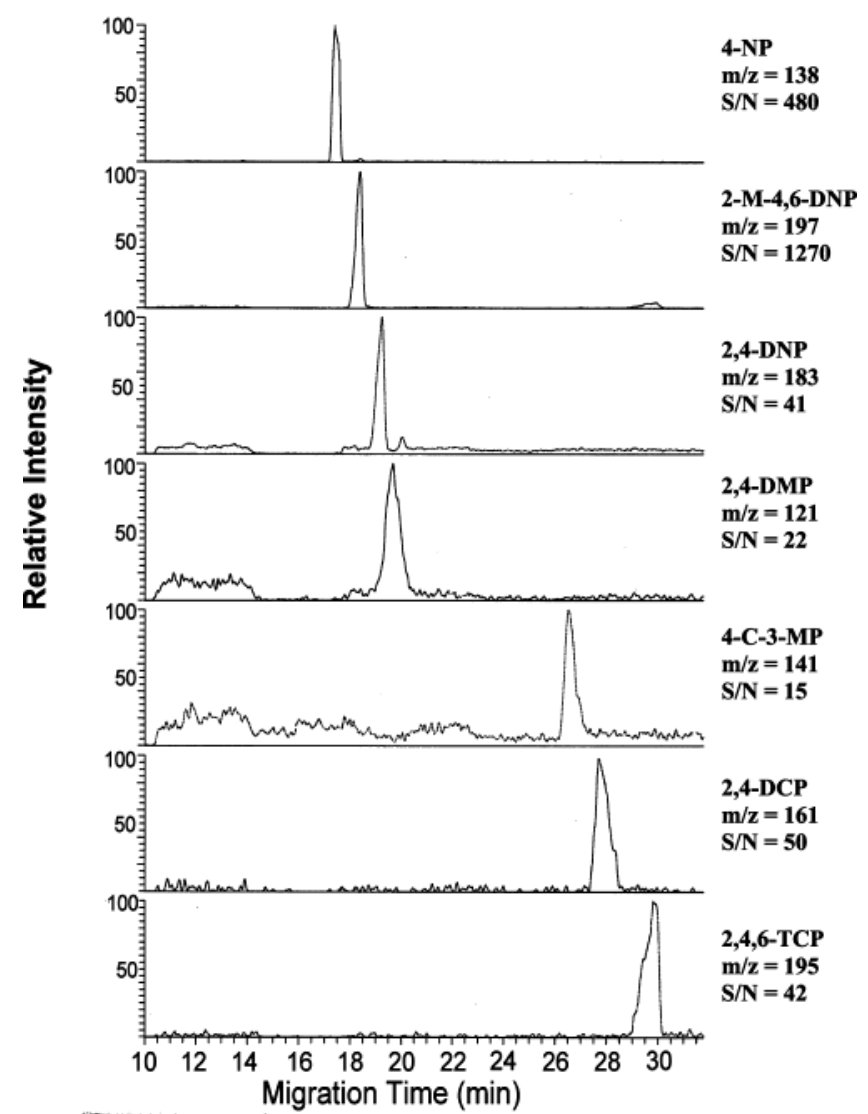

Figure 3. Mass electropherograms of $20 \mathrm{ppm}$ phenolic compounds using the low-makeup beveled tip interface in negative ion mode. The makeup liquid ( $80 \%$ 2-propanol with $1 \%$ ammonium hydroxide) was delivered at a flow rate of $400 \mathrm{~nL} / \mathrm{min}$. The potential applied to the buffer reservoir was $+20 \mathrm{kV}$, and the ESI voltage was set at $-2 \mathrm{kV}$.

Performance of the Interface in Nonvolatile B uffer CZE/ ESI-MS. In the coupling of CE with ESI-MS, nonvolatile buffers such as phosphate and borate often have significant negative effects on the ionization efficiency of the spray. ${ }^{21,22} \mathrm{~A}$ common solution to this problem is the use of volatile buffers, such as ammonium acetate, to replace the nonvolatile buffers. Unfortunately, separations obtained with more volatile buffers are often inferior to those obtained with nonvolatile buffers. For example, we have tried to analyze gangliosides, sialic acid(s)-containing glycosphingolipids, by CZE. The best result was obtained by using $50 \mathrm{mM}$ borate and $50 \mathrm{mM}$ phosphate running buffer containing $20 \mathrm{mM} \alpha-\mathrm{CD}$ at pH 9.9.23 Under the optimum conditions, the four major gangliosides (GM 1, GD 1a, GD 1b, GT 1b) were successfully separated. M oreover, each ganglioside yielded two peaks, splitting by the 28-Da difference in chain length of the ceramide moiety (Chart 1). Unfortunately, the separation obtained in CZE/ UV could not be reproduced in CE/ MS because of the suppression effect of the nonvolatile additive. Using the conventional sheath liquid interface, the concentration of borate buffer was limited to $10 \mathrm{mM}$ and only two very low abundance peaks (GDla at $\mathrm{m} / \mathrm{z}$ 918, 932) were observed (data not shown). With the use of more volatile buffers such as ammonium acetate, baseline resolution was

(21) Niessen, W. M. A.; Tjaden, U. R.; van der Greef, J. J. Chromatogr. 1993 $636,3-19$.

(22) Rundlett, K. L.; Armstrong, D. W. Anal. Chem. 1996, 68, 3493-3497.

(23) Ju, D. D.; Lai, C. C.; Her, G. R.J. Chromatogr., A 1997, 779, 195-203.

6310 Analytical Chemistry, Vol. 76, No. 21, November 1, 2004 


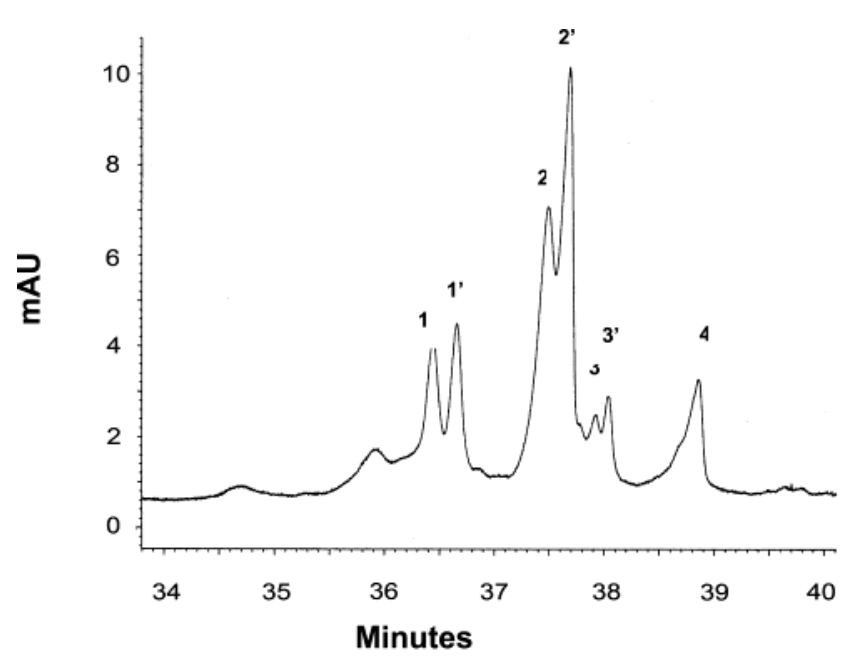

Figure 4. Electropherogram of type III gnagliosides. The separation potential was $+20 \mathrm{kV}$, and the UV wavelength was set at $200 \mathrm{~nm}$. Running buffer was $40 \mathrm{mM}$ borate and $20 \mathrm{mM} \alpha-\mathrm{CD}$ at $\mathrm{pH} 10.6$. Peaks: 1, 1', GM1; 2, 2', GD1a; 3, 3', GD1b; 4 = GT1b.

\section{Chart 1. Structures of the Gangliosides}

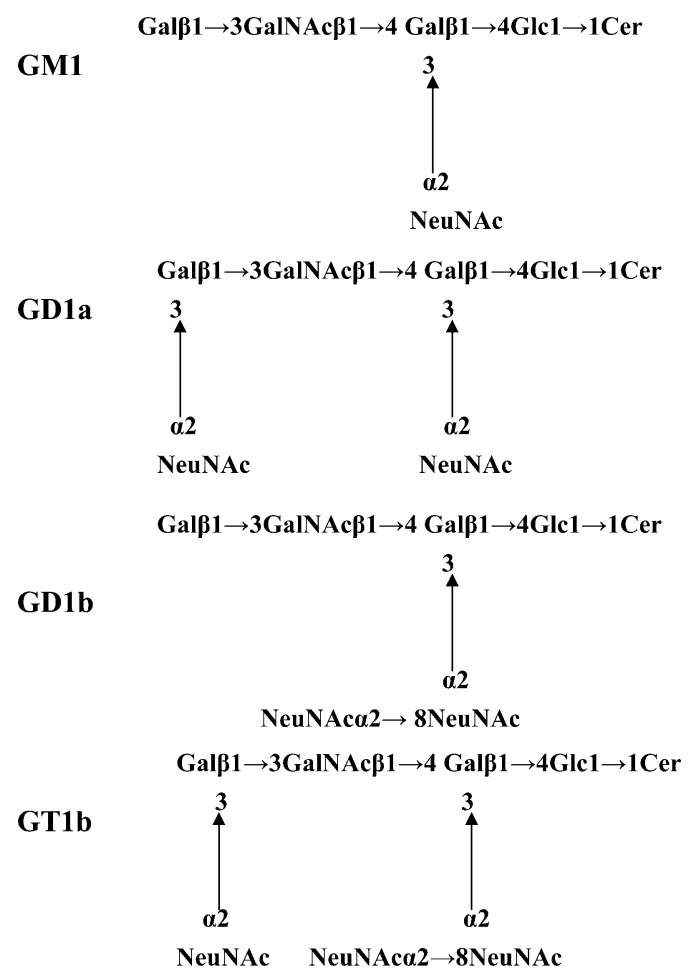

obtained for gangliosides having a different number of sugars, but the two disialogangliosides isomers, GDla and GDlb, coeluted. ${ }^{23}$

Despite the success of the low-flow flat tip interface in MEKC/ MS applications, the interface was found to be not suitable for the $\mathrm{CE} / \mathrm{MS}$ analysis of gangliosides when nonvolatile buffers were employed. Based on improved performance relative to the lowflow flat tip for MEKC/MS applications, we decided to analyze gangliosides by the low-makeup beveled tip interface. However, it is observed that the interface is not capable of handling the running buffer we used in earlier CE/ UV analysis ( $50 \mathrm{mM}$ borate, $50 \mathrm{mM}$ phosphate, and $20 \mathrm{mM} \alpha-C D$ ). Therefore, we reinvestigated the separation of gangliosides by CE/ UV. The goal was to

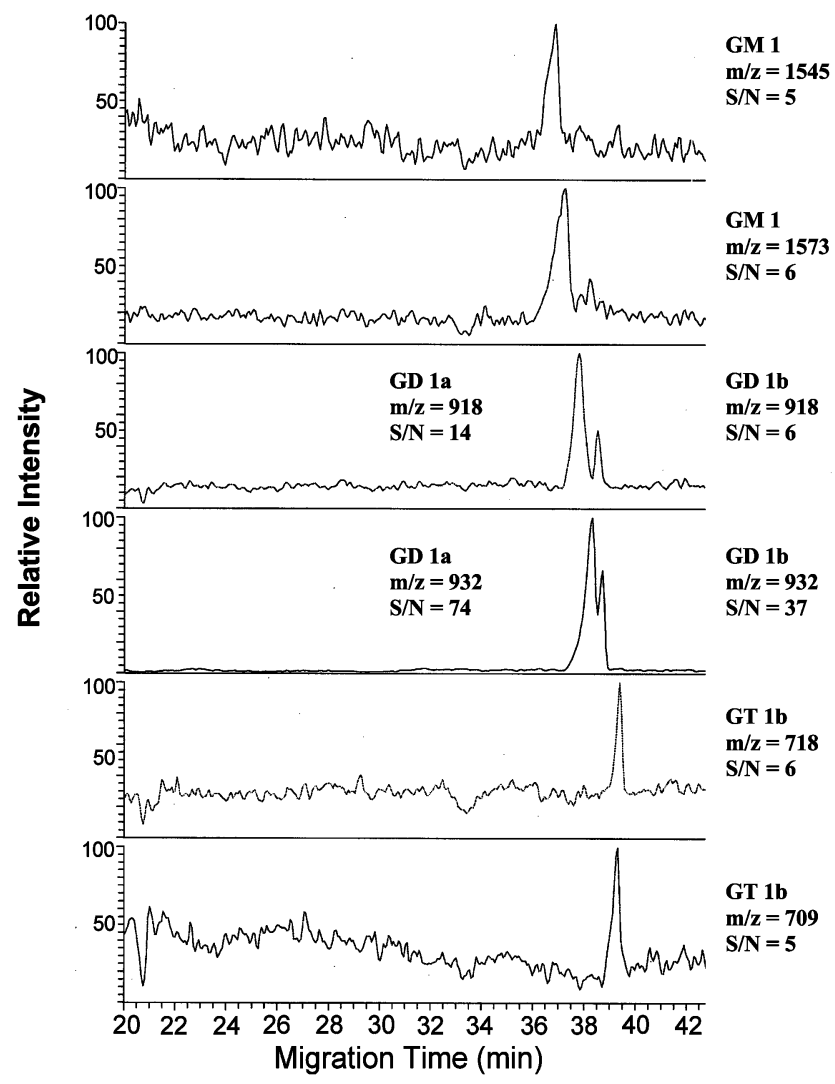

Figure 5. Mass electropherograms of $4 \mathrm{mg} / \mathrm{mL}$ ganglioside mixture using the low-makeup beveled tip interface in negative ion mode. The makeup liquid (80\% 2-propanol with $1 \%$ ammonium hydroxide) was delivered at a flow rate of $400 \mathrm{~nL} / \mathrm{min}$. The potential applied to the buffer reservoir was $+20 \mathrm{kV}$, and the ESI voltage was set at $-2 \mathrm{kV}$.

find the lowest level of buffer that could provide adequate separation. The results showed that the lowest level of buffer to provide adequate separation was $40 \mathrm{mM}$ borate and $20 \mathrm{mM} \alpha-C D$. The corresponding CE/ UV data appear in Figure 4.

Using the same ganglioside mixture, the makeup liquid composition was studied to optimize sensitivity. A solution of 2-propanol/ water (80:20) containing 1\%ammonia provided the best results. The signal intensity at different makeup flow rates was also studied. The results showed that the optimum flow rate was $400 \mathrm{~nL} / \mathrm{min}$. M ass electropherograms of gangliosides analyzed under these optimized conditions appear in Figure 5. As can be seen, the two disialoganglioside isomers (GD 1a, GD 1b) were resolved and peaks with different chain lengths were also detected for GM 1, GD 1a, and GD 1b.

\section{CONCLUSIONS}

A CE/ESI-M S interface suitable for M EKC and nonvolatile CZE analysis was developed. The interface described herein represents an extension of our previous work, which demonstrated the ability to incorporate a sheath liquid without undergoing an excessive dilution of the CE effluent. ${ }^{17}$ In contrast to the previous low-flow design, which utilized a flat tip, the present interface incorporates a beveled edge emitter. Because the present design has the same optimum flow rate, but uses a larger orifice diameter ( 75 vs 25 $\mu \mathrm{m})$, it provides similar ESI-MS sensitivity with fewer issues 
related to column plugging. Another important modification of this design is the use of a makeup column, which allows the control of the final spray solution. The analysis of triazine and phenolic mixtures demonstrates the potential of this interface for $\mathrm{MEKC/}$ ESI-MS applications in both positive and negative ion modes, respectively. Even more significant was the demonstration of $\mathrm{CE} /$ ESI-M S operation using nonvolatile buffers. Considering the fact that nonvolatile buffers are used in many CE applications, this interface provides a welcomed alternative to existing CE/ ESI-M S interfaces.

\section{ACKNOWLEDGMENT}

This work was supported by the National Research Council of the Republic of China.

Received for review May 7, 2004. Accepted August 20, 2004.

AC049330I 